\title{
Retracted: Prediction and Analysis of the Quality of Multimedia Professional Talents Combining Multiobjective Data Fuzzy Evolution
}

\author{
Advances in Multimedia
}

Received 5 November 2022; Accepted 5 November 2022; Published 22 November 2022

Copyright (C) 2022 Advances in Multimedia. This is an open access article distributed under the Creative Commons Attribution License, which permits unrestricted use, distribution, and reproduction in any medium, provided the original work is properly cited.

\begin{abstract}
Advances in Multimedia has retracted the article titled "Prediction and Analysis of the Quality of Multimedia Professional Talents Combining Multiobjective Data Fuzzy Evolution" [1] due to concerns that the peer review process has been compromised.

Following an investigation conducted by the Hindawi Research Integrity team [2], significant concerns were identified with the peer reviewers assigned to this article; the investigation has concluded that the peer review process was compromised. Therefore, we can no longer trust the peer review process, and the article is being retracted with the agreement of the editorial board.
\end{abstract}

\section{References}

[1] A. Luo and Y. Yang, "Prediction and Analysis of the Quality of Multimedia Professional Talents Combining Multiobjective Data Fuzzy Evolution," Advances in Multimedia, vol. 2021, Article ID 1623076, 7 pages, 2021.

[2] L. Ferguson, "Advancing Research Integrity Collaboratively and with Vigour," 2022, https://www.hindawi.com/post/advancingresearch-integrity-collaboratively-and-vigour/. 


\title{
Prediction and Analysis of the Quality of Multimedia Professional Talents Combining Multiobjective Data Fuzzy Evolution
}

\author{
Ao Luo ${ }^{1}{ }^{1}$ and Yongfeng Yang $\mathbb{D}^{2}$ \\ ${ }^{1}$ School of Architecture and Materials, Chongqing College of Electronic Engineering, Chongqing 401331, China \\ ${ }^{2}$ College of Geography and Tourism, Chongqing Normal University, Chongqing 401331, China \\ Correspondence should be addressed to Yongfeng Yang; 20130847@cqnu.edu.cn
}

Received 23 July 2021; Accepted 20 August 2021; Published 3 September 2021

Academic Editor: Zhendong Mu

Copyright ( $) 2021$ Ao Luo and Yongfeng Yang. This is an open access article distributed under the Creative Commons Attribution License, which permits unrestricted use, distribution, and reproduction in any medium, provided the original work is properly cited.

\begin{abstract}
In response to the relatively huge error, low efficiency, and low accuracy in the prediction and analysis data on the quality of multimedia professional talents at present, an analytical method for the prediction of the quality of multimedia professional talents based on the multiobjective data fuzzy evolutionary algorithm is proposed in this paper. The weights of the talent quality data are analyzed in detail mainly based on the multiobjective data fuzzy evolution algorithm to establish a prediction and analysis model for the quality of multimedia professional talents. This model can be used to implement the prediction and analysis of the comprehensive abilities of multimedia professional talents effectively. Finally, through a practical case study, it is verified that the method put forward in this paper has high efficiency and high accuracy in predicting and analyzing the quality of multimedia professional talents.
\end{abstract}

\section{Introduction}

Multimedia professional talents at colleges and universities are a group of professional talents who have innovative capabilities, innovative ideas, outstanding quality, and high academic attainments in academic research and discipline construction. In the cultivation of multimedia talents, a third-party certification system is applied in a timely manner as a supplementary measure to combine the third-party evaluation or social qualification certification with the multimedia teaching content [1-3]. After the students have completed the relevant courses, they will obtain a third-party certification of their professional skills through examinations or appraisal. This can provide a powerful guarantee for the subsequent internship, employment, and pursuit of further studies in the students. After the students have studied the relevant professional courses, including accepting the practical capability training, they can obtain the professional practical skills on the one hand and at the same time obtain social recognition, enter the workplace, and get a job on the other hand. This is a game-changer to the traditional mode where the teachers often do the total favor for the students instead of telling them the way to solve problems and conquer difficulties. As described in an old saying, teaching a man how to fish is better than giving him a fish, more attention should be paid to the cultivation of the innovative capability and innovative thinking of the students, focusing on the training of their practical operation skills and self-learning capacity to help the students lay a sound foundation. Thus, the corresponding teaching quality can be improved, and the practical operation capability of the students can be enhanced, so that the theory can be better combined with practice to cultivate the students more effectively. Students can not only acquire the professional technical capabilities, but also make new breakthroughs in innovation and entrepreneurship [4-6].

For the purpose of improving the comprehensive accuracy in the prediction of the quality of multimedia professional talents, a relatively long time is spent in sorting the schemes in this paper, which has effectively reduced the error rate of the system prediction and analysis. The design method of the prediction and analysis 
system for the quality of multimedia professional talents based on multiobjective data fuzzy evolutionary algorithm is applied. In the reform of multimedia technology in the cultivation of professional talents in computer science, we should insist on an overall plan and implement the plan step by step, while summarizing and making continuous improvement along with practice at the same time.

\section{Design of System Hardware}

In the design method of the prediction and analysis system for the quality of multimedia professional talents based on the multiobjective data fuzzy evolution algorithm, the visual programming language Del-phi5.0 can be adopted as the development tool [7-9]. Windows 7 is taken as the system platform to establish a prediction and analysis system for the quality of multimedia professional talents. The design method of the prediction and analysis system for the quality of multimedia professional talents based on the multiobjective data fuzzy evolution algorithm is shown in Figure 1.

2.1. Input Module. The acquisition of raw data is the main task of the input module in the prediction and analysis system for the quality of multimedia professional talents. In this paper, raw data are collected in two ways: (1) the keyboard is used to input the data in the questionnaire collected; (2) the content of questionnaire surveys is designed in the form of answer sheet, and it is input into the photoelectric reader to implement automatic identification.

\subsection{Fuzzy Comprehensive Prediction and Analysis Module.} The prediction and analysis of the quality of multimedia professional talents is implemented by using fuzzy comprehensive prediction and analysis method, and the prediction and analysis results are obtained accordingly. In the first-level fuzzy comprehensive prediction and analysis process, it is required that multiple iterations should be carried out in the fuzzy comprehensive prediction and analysis module. In the design method of the prediction and analysis system for the quality of multimedia professional talents based on the multiobjective data fuzzy evolutionary algorithm, a subprogram is designed to replace the first-level fuzzy comprehensive prediction and analysis process so as to facilitate the call by the system multiple times. The block diagram of the program is shown in Figure 2.

2.3. Report Processing Module. Reports are produced based on the results of prediction and analysis of the quality of multimedia professional talents, including a list of professional and expert quality of multimedia professional talents obtained through the prediction and analysis over the years and the ranking of the multimedia professional qualities based on their scores. The reports thus obtained can be printed and inquired $[10,11]$.
2.4. System Maintenance Module. For the purpose of preventing illegal users from entering the prediction and analysis system for the quality of multimedia professional talents without authorization and ensuring the system security and data security, a password protection function is set in the system. General users only have the browsing authority, whereas only the administrators with passwords can carry out maintenance and other tasks on the prediction and analysis system for the quality of multimedia professional talents.

\section{Method for the Prediction and Analysis of the Quality of Multimedia Professional Talents}

3.1. Establishment of Prediction and Analysis Model for the Quality of Multimedia Professional Talents. The prediction and analysis factors corresponding to the main subject of prediction and analysis are taken as elements, and the aforesaid elements are used to establish a factor set for prediction and analysis $[12,13]$. Based on the fuzzy evolutionary algorithm of multiobjective data, the prediction and analysis standard in design method of the prediction and analysis system for the quality of multimedia professional talents is obtained based on the requirements and characteristics of the prediction and analysis objects, and a factor set for the prediction and analysis of the quality of multimedia professional talents is established accordingly.

In the design method of the prediction and analysis system for the quality of multimedia professional talents based on the multiobjective data fuzzy evolution algorithm, the factor set for prediction and analysis can be divided into the first-level prediction and analysis factor set and the second-level prediction and analysis factor set. The expression of the first-level prediction and analysis factor set $U$ is shown as follows:

$$
U=\left\{U_{1}, U_{2}, \ldots, U_{m}\right\} .
$$

The first-level prediction and analysis factor set for the prediction and analysis of the quality of multimedia professional talents is further divided to obtain the second-level prediction and analysis factor set as follows:

$$
\left\{\begin{array}{c}
U_{1}=\left\{U_{11}, U_{12}, \ldots, U_{1 n}\right\}, \\
U_{2}=\left\{U_{21}, U_{22}, \ldots, U_{2 n}\right\}, \\
\vdots \\
U_{m}=\left\{U_{m 1}, U_{m 2}, \ldots, U_{m n}\right\} .
\end{array}\right.
$$

The characteristics of the factors of prediction and analysis for the quality of multimedia professional talents are combined to establish a comment set based on the expert comments and suggestions to analyze the practical situation of the prediction and analysis for the quality of multimedia professional talents. Subsequently, the quality of multimedia professional talents is classified into four grades as follows: excellent, good, medium, and poor.

In the design method of the prediction and analysis system for the quality of multimedia professional talents based on the multiobjective data fuzzy evolutionary 


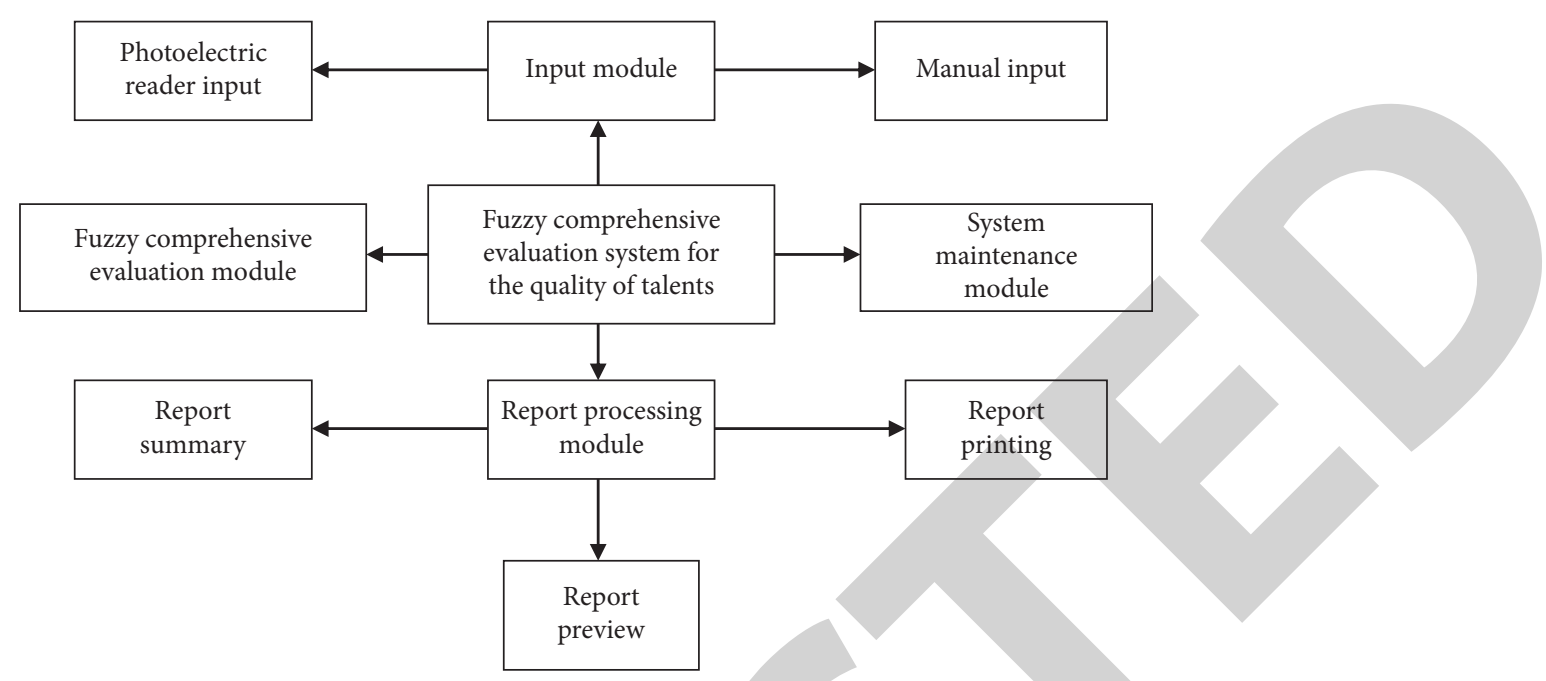

FIGURE 1: Architecture of the system for predicting and analyzing the quality of multimedia professional talents.

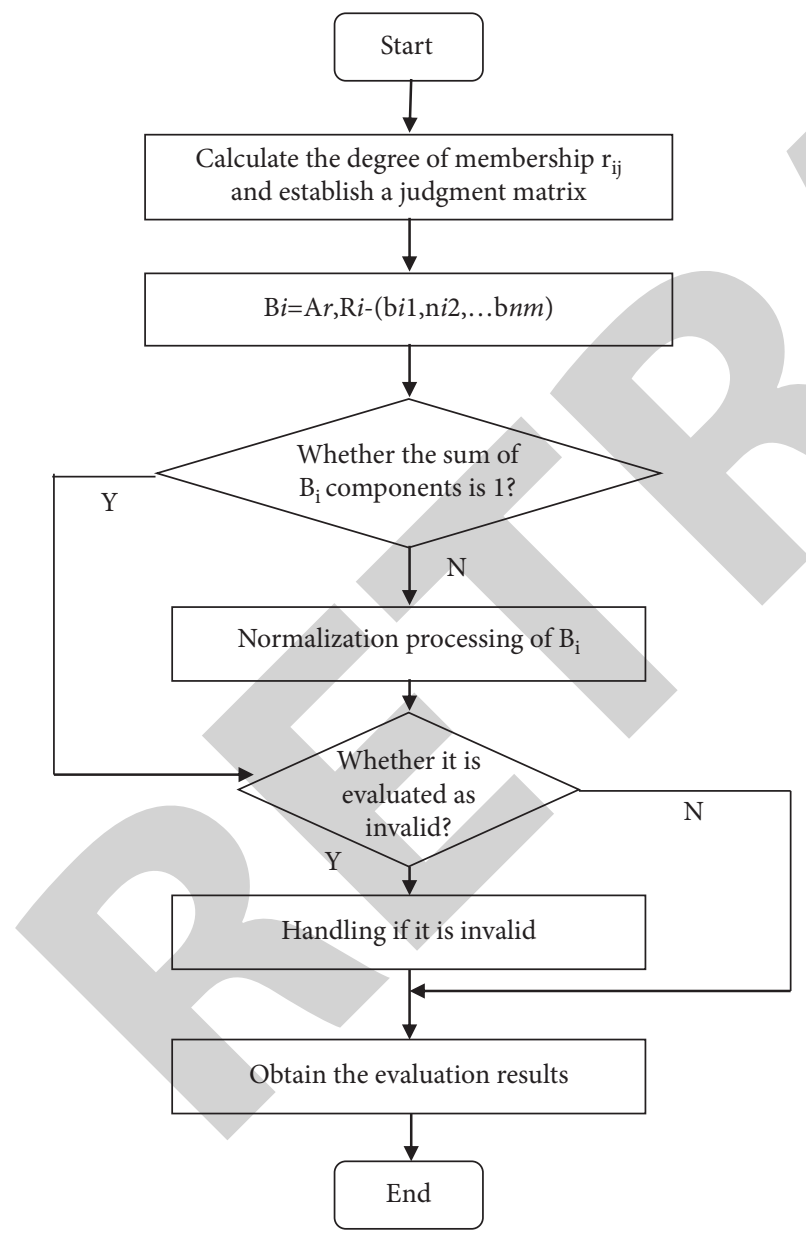

FIGURE 2: Block diagram of the prediction and analysis procedure.

algorithm, the weights of the prediction and analysis factors for the quality of multimedia professional talents are calculated through the analytic hierarchy process. The various prediction and analysis factors are divided into multiple levels based on the top-down principle, and the prediction and analysis indexes are classified according to different levels and various systems in combination with the attributes of the prediction and analysis objects $[14,15]$. Various indexes present in the same level are compared, and the standard quantification processing is carried out on the comparison results based on the corresponding importance levels to obtain the weights corresponding to the prediction and analysis indexes for the quality of multimedia professional talents. The specific steps are described as follows:

(1) The indexes existing in the same layer are compared. The judgment matrix $A$ is established, and its expression is as follows:

$$
A=\left[\begin{array}{cccc}
a_{11} & a_{12} & \cdots & a_{1 n} \\
a_{21} & a_{22} & \cdots & a_{2 n} \\
\vdots & \vdots & \vdots & \\
a_{n 1} & a_{n 2} & \cdots & a_{n n}
\end{array}\right] .
$$

The judgment matrix is normalized by column, and the following can be obtained:

$$
b_{i j}=\frac{a_{n n}}{\sum_{i=1}^{n} a_{n n}} .
$$

The judgment matrix is normalized by row, and the following can be obtained:

$$
v_{i j}=\sum_{h=1}^{n} b_{i j}
$$

It is assumed that $w_{i}$ is the weight corresponding to the quality prediction and analysis index of multimedia professional talents, and its calculation formula is shown as follows:

$$
w_{i}=b_{i j} \cdot \frac{v_{i}}{\sum_{i=1}^{n} v_{i}} .
$$

The weight vector matrix $W$ is established based on the calculated index weight of the prediction and 
analysis for the quality of multimedia professional talents.

(2) The consistency of the judgment matrix with a relatively high order is determined through the consistency test.

Based on the prediction and analysis data collected, the membership degree corresponding to the prediction and analysis index present in the prediction and analysis factor concentration is calculated, and the fuzzy matrix $U_{m n}$ is established as follows:

$$
U_{m n}=\left[\begin{array}{c}
U_{11}, U_{12}, U_{13}, U_{14}, U_{15} \\
U_{21}, U_{22}, U_{23}, U_{24}, U_{25} \\
\vdots \\
U_{m 1}, U_{m 2}, U_{m 3}, U_{m 4}, U_{m 5}
\end{array}\right] W .
$$

Here, $U_{i j}$ describes the degree of membership corresponding to the prediction and analysis factor $U_{i}$ for the comment set $U_{j}$.

Based on the calculated degree of membership, the prediction and analysis model $F$ for the quality of multimedia professional talents is established, and its expression is shown as follows:

$$
F=\frac{w_{i} \times U_{m n}}{U_{j}} \times \delta .
$$

Here, $\delta$ stands for the fuzzy operator.

3.2. Solving the Model. In the design method of the prediction and analysis system for the quality of multimedia professional talents based on the multiobjective data fuzzy evolutionary algorithm, the constructed model for the prediction and analysis of the quality of multimedia professional talents is solved by using the multiobjective data fuzzy evolution algorithm to implement the fuzzy comprehensive prediction and analysis of the quality of multimedia professional talents.

The Euclidean distance between the two weight vectors is calculated. Based on the calculation results, $T$ vectors are selected as the neighbors of the weight vector [10].

It is assumed that $B(i)=\left\{i_{1}, i_{2}, \ldots, r_{T}\right\}, i=1,2, \ldots, N$; $\lambda^{i_{1}}, \lambda^{i_{2}}, \ldots, \lambda^{i_{r}}$; plus, it describes the closest $T$ weight vectors that exist around a uniformly distributed weight vector $\lambda^{i}$.

The population $x^{1}, x^{2}, \ldots, x^{i}$ is initialized, and it is assumed that $F\left(x^{i}\right)=\left(1 / \lambda^{i_{r}}\right) F \cdot B(i)$.

The population $P$ is classified to obtain 3 subpopulations $I_{A}, I_{B}$, and $I_{C}$ so that there are $\xi_{1}$ individuals in the subpopulation $I_{A}, \xi_{2}$ individuals in the subpopulation $I_{B}$, and $\xi_{3}$ individuals in the subpopulation $I_{C}$. It is assumed that $I_{A}, I_{B}$, and $I_{C}$ in the initial stage meet the following conditions:

$$
\xi_{1}=\xi_{2}=\xi_{3}=\frac{N}{3}
$$

In the method for designing the prediction and analysis system for the quality of multimedia professional talents based on the multiobjective data fuzzy evolution algorithm, the dynamic subpopulation method is used to carry out the dynamic collaborative differential evolution. The specific process is described as follows:

(1) The new offspring individual $y^{i}$ is obtained.

(2) The reference point is updated.

(3) The evolution success rate corresponding to different strategies is calculated as follows:

$$
\begin{gathered}
\tau_{1}=\frac{\kappa_{1} / \xi_{1}}{\left(\kappa_{1} / \xi_{1}\right)+\left(\kappa_{2} / \xi_{2}\right)+\left(\kappa_{3} / \xi_{3}\right)}, \\
\tau_{2}=\frac{\kappa_{2} / \xi_{2}}{\left(\kappa_{1} / \xi_{1}\right)+\left(\kappa_{2} / \xi_{2}\right)+\left(\kappa_{3} / \xi_{3}\right)}, \\
\tau_{3}=\frac{\kappa_{3} / \xi_{3}}{\left(\kappa_{1} / \xi_{1}\right)+\left(\kappa_{2} / \xi_{2}\right)+\left(\kappa_{3} / \xi_{3}\right)} .
\end{gathered}
$$

Here, $\kappa$ stands for the evolution strategy corresponding to different individuals.

(4) The size of the population is recalculated as follows:

$$
\begin{aligned}
& \xi_{1}=N \times \tau_{1}, \\
& \xi_{2}=N \times \tau_{2}, \\
& \xi_{3}=N-\xi_{1}-\xi_{2} .
\end{aligned}
$$

Here, $\tau$ stands for the evolutionary success rate.

$\xi_{1}, \xi_{2}$, and $\xi_{3}$ are updated.

(5) Let the termination condition be $\tau>\tau_{\max }$, and the algorithm is ended. The results of the prediction and analysis model for the quality of multimedia professional talents are output to complete the fuzzy comprehensive prediction and analysis of the quality of multimedia professional talents.

\section{Experimental Results and Analysis}

For the purpose of verifying the overall effectiveness of the design method of the prediction and analysis system for the quality of multimedia professional talents based on the fuzzy evolutionary algorithm of multiobjective data, three design methods, that is, the design method of the prediction and analysis system for the quality of multimedia professional talents based on the multiobjective data fuzzy evolution algorithm, the design method of the prediction and analysis system for the quality of multimedia professional talents based on evidence reasoning, and the design method of the prediction and analysis system for the quality of multimedia professional talents based on Hall three-dimensional structure model, are adopted to carry out testing, respectively. The prediction and analysis time of the three methods in the design of the system is compared, and the test results are shown in Table 1.

Through the analysis of Table 1 , it can be known that when the system designed based on the design method of the prediction and analysis system for the quality of multimedia professional talents based on the multiobjective data fuzzy evolution algorithm is used to predict and analyze the quality 
TABle 1: Prediction and analysis time of different methods.

\begin{tabular}{lccc}
\hline Number of iterations & $\begin{array}{c}\text { Algorithm put forward } \\
\text { in this paper }\end{array}$ & $\begin{array}{c}\text { Design method for the evidence } \\
\text { reasoning system }\end{array}$ & $\begin{array}{c}\text { Design method for the Hall three-dimensional } \\
\text { structure model system }\end{array}$ \\
\hline 1 & 0.54 & 1.34 & 1.24 \\
2 & 0.67 & 1.04 & 1.27 \\
3 & 0.73 & 1.64 & 1.36 \\
4 & 0.64 & 1.38 & 1.33 \\
5 & 0.58 & 1.28 & 1.57 \\
6 & 0.55 & 1.08 & 1.33 \\
\hline
\end{tabular}

of multimedia professional talents, the time consumed is less than that consumed by the design method of the prediction and analysis system for the quality of multimedia professional talents based on evidence reasoning and the design method of the prediction and analysis system for the quality of multimedia professional talents based on the Hall threedimensional structure model to predict and analyze the quality of multimedia professional talents. In the design method of the prediction and analysis system for the quality of multimedia professional talents based on the multiobjective data fuzzy evolutionary algorithm, a subprogram is designed in the fuzzy comprehensive prediction and analysis module to replace the fuzzy comprehensive prediction and analysis process. Therefore, it is convenient for the system to call the subprogram multiple times, which has shortened the time for system prediction and analysis and improved the prediction and analysis efficiency of the design method of the prediction and analysis system for the quality of multimedia professional talents based on the fuzzy evolutionary algorithm of multiobjective data.

On the basis of the experiment described above, in the analysis of the single data and multiple data processing, the usage test results of the system CPU are shown in Figure 3.

It can be known from the data in Figure 3 that when the system put forward in this paper carries out the concurrent processing of multiple data, the usage of the CPU is increased significantly, which has demonstrated that the system put forward in this paper is more suitable for the single data analysis environment.

For the purpose of having an in-depth analysis of the statistical performance of the system designed in this paper, the quality of the multimedia professional teaching talents in local universities will be evaluated. The main implementation steps are described as follows:

(1) The evaluation object $y^{i}$ is selected

(2) The system calls the information of multimedia professional talents in the database to obtain the corresponding data and make update accordingly

(3) The evolution success rate corresponding to different strategies is calculated

(4) The relevant prediction and analysis parameters and indexes are obtained

(5) The results of prediction and analysis are generated

The method put forward in this paper can not only generate multifaceted indexes for prediction and analysis, but also give the corresponding values based on the practical

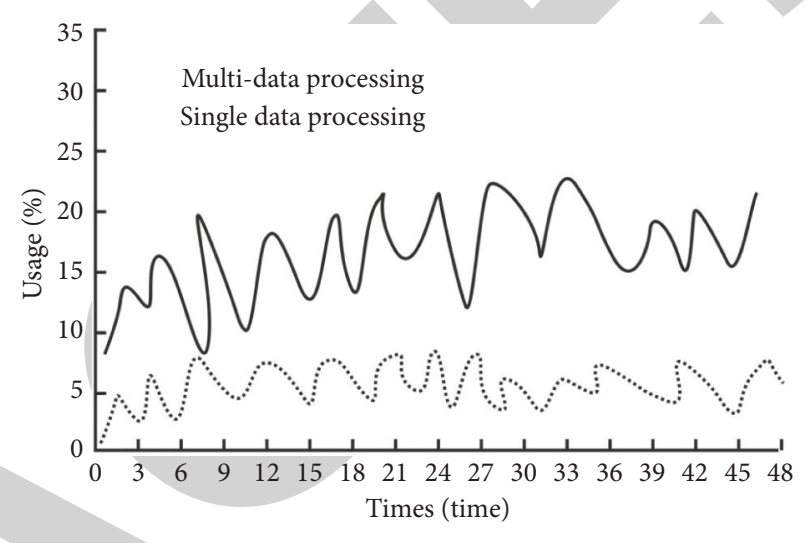

Figure 3: Analysis of CPU usage.

situation of multimedia professional talents, so it has certain feasibility. On the other hand, this multifaceted method for prediction and analysis is based on the method put forward in this paper by the application of the multiobjective data fuzzy evolution algorithm, through the analysis of multiobjective, multi-index, and various strategies, to obtain the multiobjective evolution calculation results and implement the multidimensional analysis of the prediction and analysis for multimedia professional talents.

\section{Development of Quality Prediction and Analysis Strategy}

5.1. Construction of the Target for Prediction and Analysis. The selection of a correct prediction and analysis target can fully exert the guiding, stimulating, and restricting functions of prediction and analysis. However, for the purpose of giving full play to this function, it should also be established on the basis of the full recognition of the main subject in the prediction and analysis. In the practice of prediction and analysis in higher education in our country, it is generally assumed that there is a common value system in the context of prediction and analysis. It is evident that this hypothesis can hardly solve the issue of diversification of educational value. Hence, in the establishment of a quality prediction and analysis system, special emphasis should be placed on the common "psychological construction" of the objects for prediction and analysis.

Some scholars have put forward three value orientations for the quality standards of higher education, that is, internal adaptability, external adaptability, and individual adaptability. Internal adaptability refers to the level of self- 
improvement defined within the education system; external adaptability refers to the level to which the educational services provided by higher education institutions can meet the needs of the country and society; individual adaptability refers to the level to which education can meet the active construction, promotion, and improvement of personality perfection and subjectivity of students. These three value orientations have provided ideas for designing the prediction and analysis targets for the training of the quality of multimedia professional talents. Through discussion and exploration, the subjects of prediction and analysis can realize that the target of quality prediction and analysis is to understand the level and status of the training quality of professional talents in line with these three value orientations and understand the problems present in the training of professional talents from the analysis of the level and status as well as the improvement measures. The coestablishment of prediction and analysis targets is to form a common prediction and analysis vision for the predictive analysts and predicted analysts, thereby paving the way for the effective development of the prediction and analysis activities.

5.2. Negotiation of the Prediction and Analysis Scheme. The design of the prediction and analysis scheme is an essential task in the process of establishing a prediction and analysis system. It is necessary to guide various prediction and analysis subjects to have extensive negotiation and strive to design scientific, reasonable, and highly operable prediction and analysis indexes, thereby laying a sound foundation for the overall prediction and analysis work.

In general, the index design of a number of prediction and analysis schemes is carried out based on the concept of "resource index, process index, and performance index." In the design of the quality prediction and analysis index scheme, the whole prediction and analysis scheme can be set in four aspects, that is, "talent training targets and plans," "development and utilization of talent training resources," "talent training process and control," and "talent training performance" based on basic criteria of "highlighting professional characteristics," "highlighting talent training performance prediction and analysis," "emphasizing both quantitative and qualitative prediction and analysis at the same time," and so on. The index of "talent training targets and plans" is set based on the characteristics that higher vocational majors should be market-oriented and serve the regional economy so as to allow the higher vocational professional talent training targets to be more in line with the market demand for talents and strengthen the demonstration of the scientific nature of talent training programs. In the two indexes of "development and utilization of talent training resources" and "talent training process and control," "resource indexes" and "process indexes" are emphasized as well as "utilization" and "control"; that is, quality management is emphasized. The prediction and analysis of "talent training performance" is the focus of the quality prediction and analysis index program. It is carried out to determine the overall quality, capability of seeking employment, and corporate satisfaction of higher vocational graduates, as well as the future contribution rate and influence on professional positions. Hence, it is an essential basis for the determination of the talent training targets and programs.

5.3. Open Prediction and Analysis Channels. In the practice of quality prediction and analysis, various subjects of prediction and analysis are relatively scattered, independent, and with their respective characteristics. Hence, it is necessary to adopt open and convenient channels and approaches to allow the majority of stakeholders to fully participate in prediction and analysis. The realistic and reliable approach at present is to carry out prediction and analysis based on the use of network platforms, that is, to design prediction and analysis questionnaires, develop prediction and analysis software, and then guide and organize predictive analysts to participate in the network prediction and analysis. Network prediction and analysis is relatively convenient, and there is no exact time limit, which is conducive for the participants to have a deep understanding, analyze the problems more thoroughly, and put forward strategies and suggestions for addressing the problems and improving the situation accordingly; it is also very easy to organize, with a relatively low operating cost, and convenient for data statistics, analysis of the causes, making summaries, and drawing conclusions accordingly.

It should be noted that, in the organization of prediction and analysis activities, it is unrealistic to expect that all stakeholders can be fully involved in the prediction and analysis. The reason is that prediction and analysis is always subject to the constraints of time and funding. Hence, as the main subject of the prediction and analysis organization, it should seek the optimization of the involvement of stakeholders in the prediction and analysis as much as possible. In addition, prediction and analysis methods such as forums, individual interviews, data access, and field visits are also indispensable in the overall prediction and analysis work. Through these methods, it will be more conducive for higher vocational majors to grasp the key issues and valuable improvement measures in the training of talents, as well as the in-depth thinking of the stakeholders accurately.

\subsection{Exchange of the Prediction and Analysis Information.} Without high-quality information processing, there will be no effective improvement and continuous advancement of prediction and analysis objects. In the prediction and analysis of the training quality of higher vocational talents at present, every step and each link in the information collection and processing seems to be accurately designed and planned. However, the statistical results of prediction and analysis are often distorted or biased, or the issues are not disclosed and responded in a timely and effective manner. Hence, in the construction of a quality prediction and analysis system, it is necessary to attach great importance to the processing of prediction and analysis information and strive to overcome the tendency to seek only obtaining the quantitative results of prediction and analysis, while ignoring the communication with and disclosure to predictive 
analysts. In fact, in the whole prediction and analysis activity, whether it is in the collection, screening, confirmation, or processing of information, senior vocational personnel will be in a state of information exchange with the participants involved in prediction and analysis. Thus, the communication and feedback of information between higher vocational majors and various prediction and analysis subjects run through the key links of the whole prediction and analysis process. The continuous state of exchange and communication between the aforesaid two can not only ensure that the results of prediction and analysis are fully open to the stakeholders, but also ensure that higher vocational professional talents can understand their true status and timely identify and improve their deficiencies accordingly.

\section{Conclusions}

As a key link in the current teaching activities at colleges and universities, the prediction and analysis of the quality of multimedia professional talents can effectively identify whether the quality of the multimedia professional talents meets the talent standards in theory. In this paper, the multiobjective data fuzzy evolutionary algorithm is introduced into the process of prediction and analysis for the quality of multimedia professional talents, which can quickly accomplish the prediction and analysis of the quality of multimedia professional talents in a short time and lays a sound foundation for the development of the prediction and analysis system for the quality of multimedia professional talents.

\section{Data Availability}

The data used to support the findings of this study are available from the corresponding author upon request.

\section{Conflicts of Interest}

The authors declare that there are no conflicts of interest.

\section{Acknowledgments}

This research study was sponsored by Chongqing Postgraduate Education and Teaching Reform Research Project ("Study on the Reform and Practice of the Education System of "Explicit Ability" of Masters in Tourism Management," under project number YJG182020, and "Study on the Improvement of Education Quality of Postgraduate in Geography Based on the OBE Concept," under project number YJG203062). This work was also supported by Humanities and Social Sciences Research Projects of Chongqing ("The Enlightenment of American Student Affairs System to Chinese Student Management, under project number 17SKG042). The authors acknowledge the support of these projects.

\section{References}

[1] I.-W. G. Kwon, S.-H. Kim, and D. G. Martin, "Healthcare supply chain management; strategic areas for quality and financial improvement," Technological Forecasting and Social Change, vol. 113, no. 2, pp. 422-428, 2016.

[2] E. Johannesson and J. Skogsborg, "Talent predictioning sett utifrån MOA-modellen," Economics \& Business, vol. 1, no. 5, pp. 42-48, 2017.

[3] I. R. Faber, M. T. Elferink-Gemser, F. G. J. Oosterveld, J. W. R. Twisk, and M. W. G. Nijhuis-Van der Sanden, "Assessing personal talent determinants in young racquet sport players: a systematic review," Journal of Sports Sciences, vol. 34, no. 5, pp. 1-16, 2016.

[4] S. Bertoli, V. Dequiedt, and Y. Zenou, "Can selective immigration policies reduce migrants' quality?” Journal of Development Economics, vol. 119, no. 3, pp. 100-109, 2016.

[5] V. Rojenko and A. Dahs, "Demographic determinants of creativity: the analysis of the development of creative potential and forecast for the Baltic states," Economics and Business, vol. 30, no. 1, pp. 79-90, 2017.

[6] D. Bates, "Tunes, tubes and clouds: getting your in-house talent out of the house and online. Part 1: production," Legal Information Management, vol. 16, no. 1, pp. 44-48, 2016.

[7] J. Berke, R. Szabó, I. Bérczy et al., "Digital photo school student workshop possibilities develop talent," Journal of Applied Multimedia, vol. 3, no. 2, pp. 36-40, 2015.

[8] R. Geetha, K. Ramyadevi, and M. Balasubramanian, "Prediction of domestic power peak demand and consumption using supervised machine learning with smart meter dataset," Multimedia Tools and Applications, vol. 3, no. 1, pp. 1-19, 2021.

[9] N. E. Mamonto, H. Maulana, D. Y. Liliana et al., "Multimedia content development as a facial expression datasets for recognition of human emotions," IOP Conference Series: Materials Science and Engineering, vol. 306, no. 2, pp. 1090-1102, 2018.

[10] A. Etinkaya and M. K. Baykan, "Prediction of middle school students' programming talent using artificial neural networks," Engineering Science and Technology: An International Journal, vol. 2, no. 1, pp. 1-9, 2020.

[11] D. Shen, J. Liu, D. Li et al., "Evaluation model and empirical research on the contribution rate of meteorological talents," Microprocessors and Microsystems, vol. 2, no. 5, pp. 10-19, 2021.

[12] R. Ding, C. H. Muntean, and G.-M. Muntean, "Energy-efficient device-differentiated cooperative adaptive multimedia delivery solution in wireless networks," Journal of Network \& Computer Applications, vol. 5, no. 3, pp. 56-63, 2015.

[13] X. Chen and T. Wang, "General aircraft material demand forecast based on modified PSO optimized BP neural network," International Journal of Multimedia and Ubiquitous Engineering, vol. 11, no. 6, pp. 181-194, 2016.

[14] B. Xu, X. Li, L. Hao et al., "Research on professional talent training technology based on multimedia remote image analysis," EURASIP Journal on Image and Video Processing, vol. 9, no. 1, pp. 1-9, 2019.

[15] P. Ilango and P. J. Kumar, "MQRC: QoS aware multimedia data replication in cloud," International Journal of Biomedical Engineering and Technology, vol. 25, no. 4, pp. 250-259, 2017. 